\title{
Multicast Routing Algorithm Based On Genetic Algorithm
}

\author{
Yanhua Chen \\ Modern Education Technology Center, Neijiang Normal University, \\ Neijiang 641100, China \\ Cyhzxb@126.Com
}

\begin{abstract}
MRAGA (Multicast Routing Algorithm based on Genetic algorithm) for the DelayConstrained Minimum-Energy Multicast Routing problem was presented. The genetic operators of this algorithm reduce the transmission delay and energy consumption of multicast trees, thus accelerating the convergence speed of the algorithm. Experiment results show that the multicast tree found by this algorithm not only guarantee the delay constraint, but also has the minimum energy consumption. Furthermore, this algorithm converges quickly.
\end{abstract}

Keywords: Wireless Ad Hoc network, QoS routing, Multicast, Genetic algorithm

\section{Introduction}

Multicast routing technology based on delayed inhibition has great significances to network real-time multimedia application [1-2]. Most of multimedia applications have strict delay constraints of network, even requiring live transmission. Besides, how to reduce energy consumption is one of key problems with Ad Hoc network [3-9]. At present in Ad Hoc network, studies on QoS multicast routing refer largely to such problems as delay-constrained least-cost multicast routing, bandwidth delay-inhibited minimal cost multicast routing, as well as delay and delay jitter constrained minimal cost multicast routing. Those researches didn't consider how to cut down energy dissipation while looking for the path which can meet QoS restrictive condition. Hence their proposed algorithms can't be used directly for Ad Hoc network. Additionally, plenty of scholars have discussed from the perspective of Ad Hoc network about how to utilize effectively the limited energy of it. Valuable achievements have been made; however, they overlooked delay constraints.

For the above reasons and the importance of delay-constrained least energy consumption multicast network to Ad Hoc network, we introduce delay inhibited least energy consumption multicast routing problem and define it. Further on, we propose one new genetic algorithm to address it. Delay constrained least energy consumption multicast tree guarantees the transmission delay of any node from source to any destination not exceeding the given upper limit and the tree consumers the least energy. Thus, Ad Hoc network energy is saved in the meanwhile of ensured multicast business service quality (delay). In Ad Hoc network, if network application has higher delay requirement, like video conference and sensor network real-time data monitoring, it's advisable to apply delay inhibited least energy consumption multicast routing algorithm for multicast communications, which not only suffices delay inhibition and optimizes total energy consumption. 


\section{Problem Presentation}

\subsection{Creation of Network Model}

Suppose one wireless Ad Hoc network with N nodes. Each node has unique identifier i, $1 \leq i \leq N$ and network connectivity depends on the transmission power of each node. In the network, each node can adjust dynamically their sent energy. When one node joins in several multicast tasks, it can choose different emitted energy for varied multicast trees as to transfer data packets.

To simplify, we assume here all data packets of the same size. Suppose network topology is changeable, but it's not changing frequently to the extent where routing computation becomes invalid. Precisely, suppose there is at least one stable period after topological change. Also assume all nodes in wireless Ad Hoc network using Omnidirectional antenna. Compared with wired network, wireless Ad Hoc network has the advantage of wireless multicasting [10-13], i.e., when one node sends one packet, all nodes in its transmitting power coverage area can receive the packet. Besides, each node $\mathrm{i}$ has two coverage areas, which are:

(1) Control coverage area, credited as $C R_{i}$

(2) Data coverage area, credited as $D R_{i}$

Control coverage area and data coverage area are dependent separately on the emitted energy by node i used for sending control packet and data packet.

\subsection{Creation of Energy Consumption Model}

Energy consumption model on one wireless link was elaborated in [14-15]. The typical radio communication energy attenuation model is $\beta$, where $\beta$ is path loss factor, constant related to radio communication environment. Based on the radio communication energy attenuation model, when one data packet is sent from node $v_{i}$ to node $v_{j}$, the energy used up at node $v_{i}$ is:

$$
E_{i, j}=k\left(l_{i, j}\right)^{\beta}+E_{o}
$$

Where $l_{i, j}$ is the Euclidean distance between the nodes $v_{i}$ and $v_{j}, k$ is and antenna characteristics are related to the constant, $E_{0}$ is receiving a packet of data to the consumption of energy.

\section{Multicast Routing Algorithm Based on Genetic Algorithm (MRAGA)}

Figure 1 shows MRAGA algorithm. The input of it is Ad Hoc network topology G, source node $s$ and destination node collection D. Chromosome (i) is individual i in current population. MSTSelect() is selection operator. $T_{a}, T_{b}$ and $T_{c}$ are individuals; $\operatorname{Rand()}$ is a function, generating random figure between $[0,1]$. Crossover() and Mutation() refer to crossover operator and mutation operator. $N_{p}$ is population size. $N_{p}$ is size of population. $N_{\text {optimal }}$ is number of optimal individuals, $p_{c}$ is cross probability and $p_{m}$ is mutation probability. RandomDFS() is random depth first search algorithm.

\subsection{Encoding}

The encoding method of multicast tree affects greatly the performance of genetic algorithms. So far, representative multicast encoding methods are 1D binary encoding, Prüfer number encoding and ST encoding. However, those methods produce illegal multicast trees in genetic procedure like ST encoding, or do bad in location inheritance 
like Prüfer encoding, or degrade the efficiency of genetic algorithms when the search space expands sharply because of bigger network size. We suggest using multicast tree to describe individuals (chromosomes) in genetic space.

\subsection{Population Initialization}

In initializing the population, it's necessary to consider two questions:

(1) Population scale, marked as $N_{p}$;

(2) The method for producing each individual in the population

$N_{p}$ needs appropriate selection. If it's too small, the genetic algorithm will search out local optimal solution; if it's too big, the genetic algorithm will work inefficiently. When the algorithm is designed well, $N_{p}$ can be set a proper value by pre-testing.

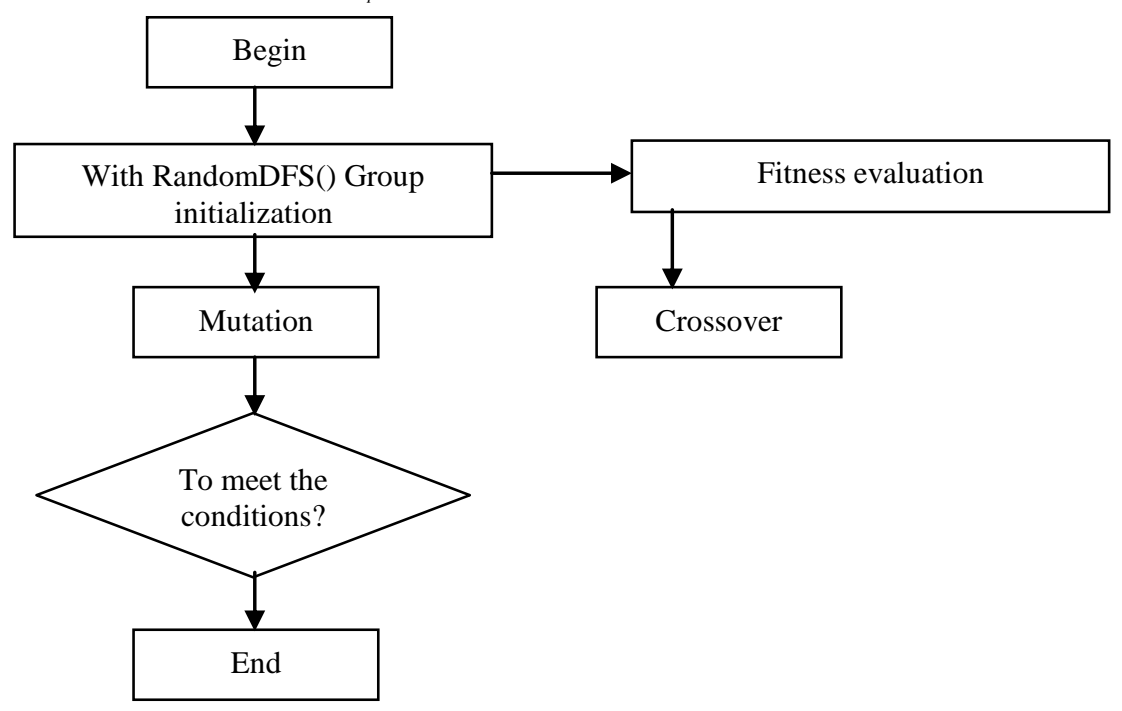

Figure 1. Flowchart of MRAGA

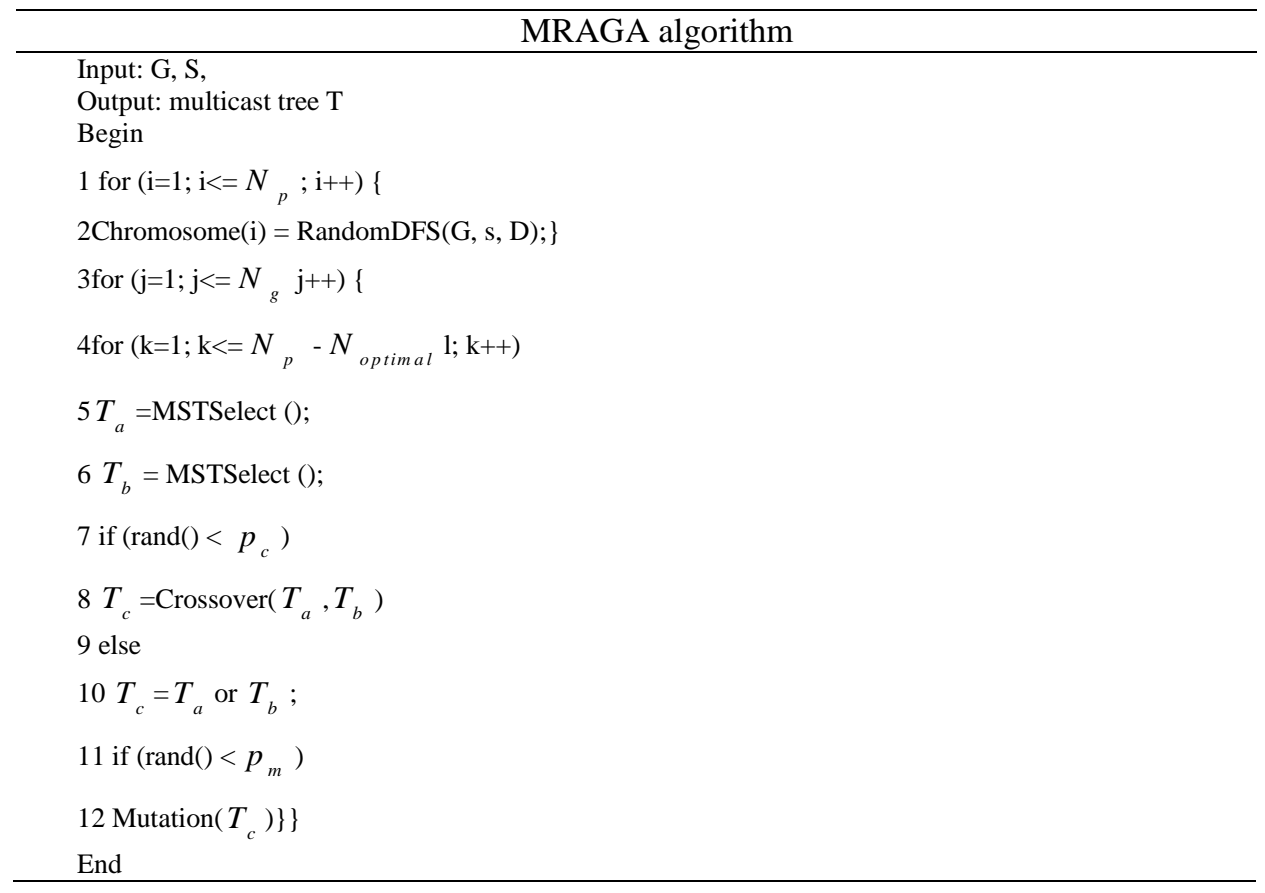

Figure 2. Peudo Code of MRAGA 


\subsection{Fitness Function}

Fitness function reflects accurately goodness or badness of individual character (i.e., solution). The individual with good characters has higher fitness. Delay-Constrained Minimum-Energy Multicast Routing problem is to look for multicast tree which meets with delay constraint and consumes least energy. So the multicast tree adequate for both conditions has higher fitness. The fitness function is defined like:

$$
f(T)=\frac{a}{E C(T)} \prod_{V_{t} \in D} \Phi\left(\operatorname{delay}\left(p_{T}\left(s, v_{t}\right)\right)-\delta\right)
$$

\subsection{Selective Operator}

In MRAGA algorithm, it adopts elitist model as selective operator. With the selection model, we can pick up the best individuals (ones with highest fitness, about $N_{\text {best }}$ of them) and reproduce them directly to the next population. Then, from the rest individuals in the current population, we choose by roulette mechanism father individuals for crossover operation. Individual $i$ is chosen at the probability:

$$
p\left(T_{i}\right)=\frac{f\left(T_{i}\right)}{\sum_{j=1}^{N_{p}} f\left(T_{j}\right)}
$$

\subsection{Crossover Operator}

By roulette mechanism, we choose one pair of individuals as parent. Crossover operator performs crossover operation according to crossover probability $p_{c}$, in order to produce one offspring individual. Use $T_{a}$ and $T_{b}$ to represent the pair of parent individuals; $T_{c}$ means offspring individual cross-produced by $T_{a}$ and $T_{b}$. Crossover operator inherits links and multicast groups (i.e., source nodes and all destination nodes) shared by $T_{a}$ and $T_{b}$ to offspring individual $T_{c}$. According to the definition of fitness function and selection operator, better individuals (with higher fitness) are more possibly chosen as parent individuals. Therefore, links shared by parent individuals suggest more probably "good" character or nature of individuals. Handing down multicast groups to offspring individual $T_{c}$ is to guarantee all multicast group members included in new individuals. However, shared links and multicast groups inherited to offspring individuals will result in some separate sub-trees in $T_{c}$. So it's required to choose some links with which those separate trees connect to one legal multicast tree. It is shown in Figure 3.

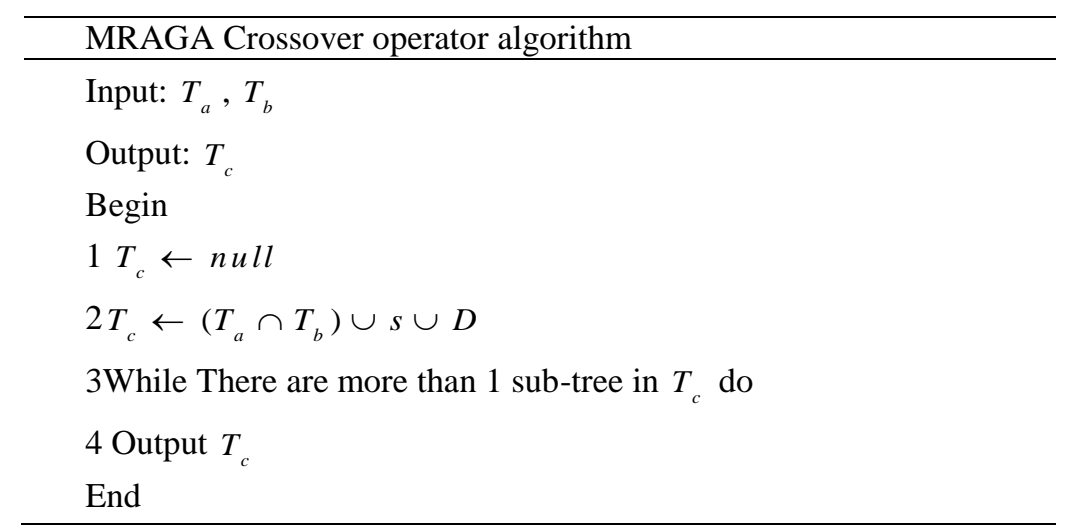

Figure 3. Pseudocode of MRAGA Crossover 


\subsection{Mutation Operator}

After one new individual is generated, mutation operator performs mutation operation according to mutation probability $p_{m}$, in the following steps:

Step 1 Choose randomly one group of nodes from individuals;

Step 2 Remove from individuals the edges between this group of nodes and their father nodes, producing some isolated sub-trees among them;

Step 3 Use the shortest path connection mechanism or shortest delay linking mechanism to join up those isolated sub-trees to form one new multicast tree. It is shown in Figure 4.

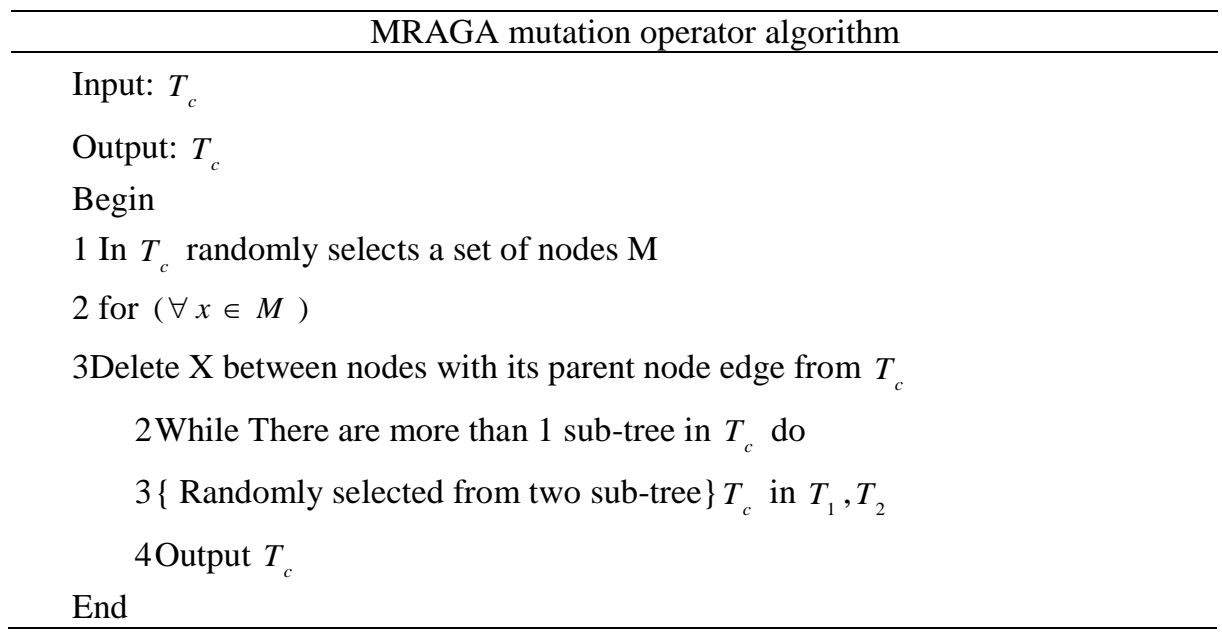

Figure 4. Pseudocode of MRAGA Mutation

\section{Comparisons with Other Algorithms}

Here we compare MRAGA with two other multicast routing algorithms with best effects. They are Haghighat's GA (HGA) [16] and Yen's GA (YGA) [17].

\subsection{Coding Way}

HGA adopts Prüfer number coding way. This way has bad location inheritance. In the procedure of inheritance, illegal multicast trees will be produced, requiring examination and restoration.

YGA adopts ST encoding way. This method can lead to illegal multicast trees and routing loop during inheritance, requiring examination and restoration.

MRAGA uses tree-like coding method, using multicast tree to represent individuals. Tree-like encoding mechanism omits encoding and decoding in the genetic process, simplifying genetic algorithms. This encoding mechanism overcomes the weakness of Prüfer number coding way. Based on tree-like encoding way, we design properly crossover operator and mutation operator, avoiding the production of illegal individuals and routing loops in inheritance. Thus, no need of additional check-out and restoration simplifies the genetic algorithm.

\subsection{Selection Model}

HGA and YGA adopt roulette selection model. This model may eliminate the best solution in the genetic process, making convergence speed down or not converge.

MRAGA adopts elitist method and roulette selection model to complete the selective process. Prior to crossover and mutation operation, we need to reproduce directly the best individuals in current population to the next generation, to guarantee the optimal units of 
one generation not damaged; then, use roulette model to choose father individuals for crossover operation, making individuals with higher fitness inherit more possibly their excellent features to the next generation and making the genetic algorithm converge to the optimal solution.

\subsection{Crossover Operator}

HGA has bad location inheritance in crossover process. Father individuals' good natures are hardly passed down to the next generation. And in the process, it will cause infeasible solution.

In crossover process, YGA will produce routing loop and infeasible solutions.

MRAGA's crossover operator overcomes the weakness of HGA. Father individuals' good characters can bring down to the next generation by their shared links. Also in crossover operation, MRAGA won't produce routing ring and unlawful individuals. Hence, it requires no extra examination and restoration. MRAGA takes into account delay and distance, promoting the convergence speed of GA.

\subsection{Mutation Operator}

In the mutation operation, YGA and HGA introduce new individuals to the population, making GA not fall into local optimal solution.

Comparatively, MRAGA introduces not only new individuals, avoid falling into local optimal solution; and also considers delay and distance, optimizing delay and energy consumption and increasing convergence speed of GA.

\section{Experiment Design and Discussion}

\subsection{The Experimental Setup}

The proposed algorithm is realized with the use of $\mathrm{MS} \mathrm{VC}++6.0$, together with Genetic Algorithmlib (GAlib) [18]. GAlib is one GA C++ library. The experiment environment is one PC with Pentium dual core $2.5 \mathrm{GHz}$ CPU (2GB RAM). To make HGA and YGA applicable for Delay-Constrained Minimum-Energy Multicast Routing problem, in the implementation of HGA algorithm, we don't consider bandwidth inhibition, i.e., in our tested network, we think these networks satisfy the bandwidth inhibition.

Simulation tests are carried out in different random networks, whose size covers 20100 nodes. Since the current Ad Hoc network is mainly used in small to medium scenarios like military field, disaster rescue, mobile conferencing, which have tens of nodes, so tests on network with 20-100 nodes have practical meanings. For networks with specific scale (fixed number of nodes), nodes are randomly distributed. Each node lies in at least one-node control coverage area, i.e., network is connected. Link delay is evenly distributed in $[0,50]$; link distance evenly in $[10,200]$. Each routing request includes three parameters: source node s, all destination node collection $\mathrm{D}$ and maximum delay $\delta$. Regarding each routing request, the three parameters are randomly generated. The maximum delay $\delta$ is evenly distributed in $[30,160]$.

For one network with specific size, experimental results are acquired based on 1000 randomly generated networks, of which 10000 routing requests are produced in each network. In the experiment, other parameters set as follows: in energy consumption model, parameter $\mathrm{k}=1, E_{0}=1, \beta=2$. Through prior implementation tests, we find MRAGA performs well when $p_{c}=1, p_{m}=0.05, N_{p}=15$. All algorithms end after 5000 individuals are produced. 


\subsection{Performance Evaluation Indicators of Algorithms}

We tested algorithms' performance from three aspects: routing success ratio (SR), energy cost ratio and running time. Routing SR describes whether the multicast tree found by the algorithm meets delay constrained condition; energy cost ratio describes the energy consumption of multicast tree found by the algorithm; running time involves with the algorithm's convergence speed.

\subsubsection{Routing Success Ratio}

Routing success ratio is defined like:

$$
S R=\frac{\text { The number of routing request }}{\text { The total number of request routing }}
$$

For a routing request, an algorithm to construct a multicast tree if meet the delay constraints is considered the routing request success.

\subsubsection{Energy Cost Ratio}

For a given network legend and routing request, the energy consumption of multicast tree constructed by the algorithm i is put as EC. As currently no algorithm can find the least energy consumption of delay constrained least energy consumption multicast tree, EC ratio is employed instead. It's defined as:

$$
\text { Energy cost ratio algorithm } \mathrm{i}=\frac{E C_{i}}{\min \left\{E C_{H G A}, E C_{Y G A}, E C_{\mathrm{MRAGA}}\right\}}
$$

\subsubsection{Running Time}

In the search process of GA, it refers to the time consumed during population evolving to a stable state. Shorter running time means less time used by GA for evolving to stable state (i.e., search the best solution) and quicker convergence speed.

\subsection{Routing SR Comparison}

Figure 5 offers routing SR results of HGA, YGA and MRAGA. Least distance technique (LDT) has the highest routing SR for it uses the shortest delay path to connect source node and each destination node. So we compare the three algorithms and LDT in terms of routing SR. LDT's routing SR offers reference points.

From the picture5, we see HGA, MRAGA and LDT have similar routing SR and their routing SRs are all higher than YGA's. That is because HGA and MRAGA consider delay factor in crossover operation, while YGA doesn't do so. Our findings demonstrate that as long as there exist multicast trees which meet delay constrained condition, HGA and MRAGA can find them. 


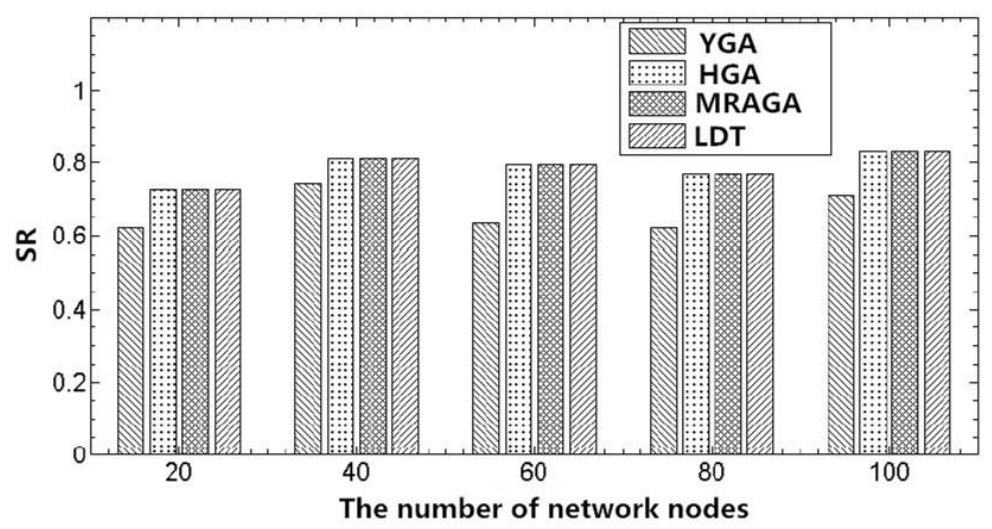

Figure 5. Comparison of SR (Multicast Group Size: 20\%-30\% Network Nodes)

\subsection{Comparison of Energy Consumption}

Figure 6 and 7 show respectively energy cost ratio when multicast group's node number is $20 \%$ and $30 \%$ of network nodes. As seen from them, MRAGA reaches the least EC ratio, approximating 1. Both HGA and YGA's EC ratio is bigger than MRAGA. In short, compared with HGA and YGA, energy consumption of multicast trees constructed by MRAGA is the least, for the reason of its consideration of distance factor in crossover and mutation operations.

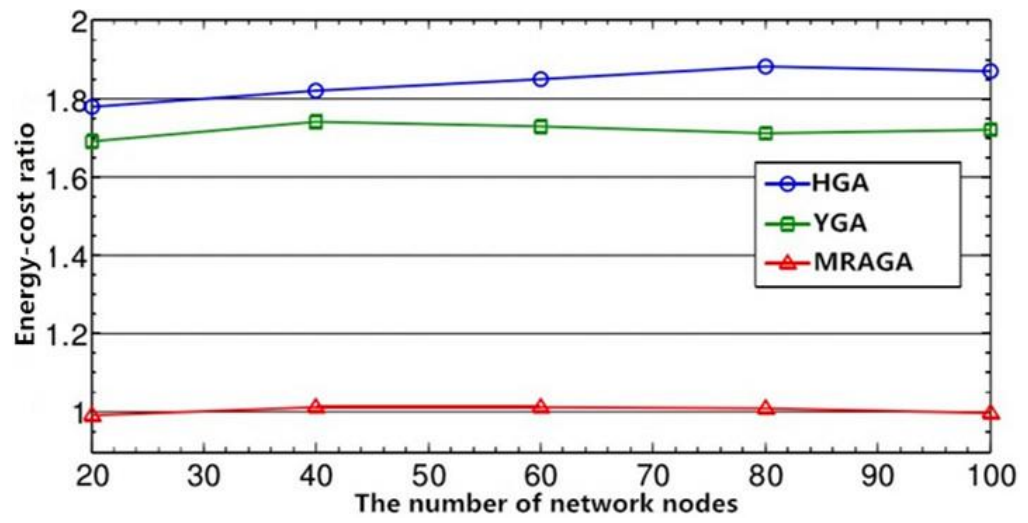

Figure 6. Comparison of Energy-Cost Ratio (Multicast Group Size: 20\% Network Nodes)

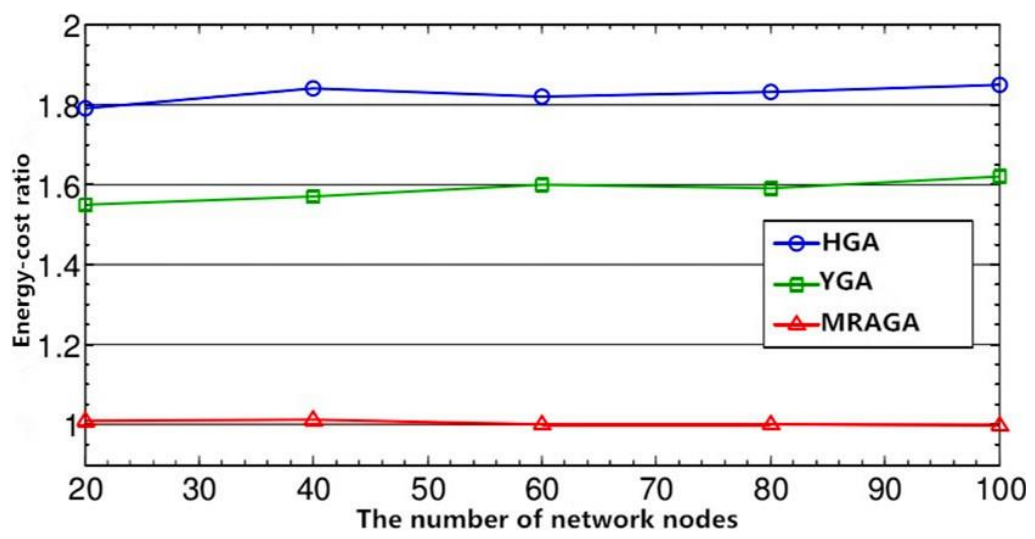

Figure 7. Comparison of Energy-Cost Ratio (Multicast Group Size: $\mathbf{3 0 \%}$ Network Nodes) 


\subsection{Comparison of Running Time}

Figure 8 presents running time results when multicast group's node number is $20-30 \%$ of network nodes. From Figure 8, we see MRAGA runs the shortest time; also its running time grows slowly with aggrandizing network size. HGA and YGA run longer than MRAGA. When network scale is over 60 nodes, HGA and YGA's running time increases rapidly. Experimental results confirm that MRAGA runs the shortest time of them three GAs. The shorter time the algorithm takes to tend to be stable (find the optimal solution), the quicker the convergence speed becomes.

\section{Conclusion}

To solve the problem of Delay-Constrained Minimum-Energy Multicast Routing proposed a new crossover operator and mutation operator. In the crossover operator and mutation operator consider the delay and distance, can reduce the group multicast tree delay and energy consumption, accelerate the speed of convergence of the MRAGA. Experimental results show that MRAGA algorithm to construct a multicast tree not only satisfies the delay constraints, and multicast tree energy consumption is the most minimal, and the convergence speed MRAGA algorithm is fastest.

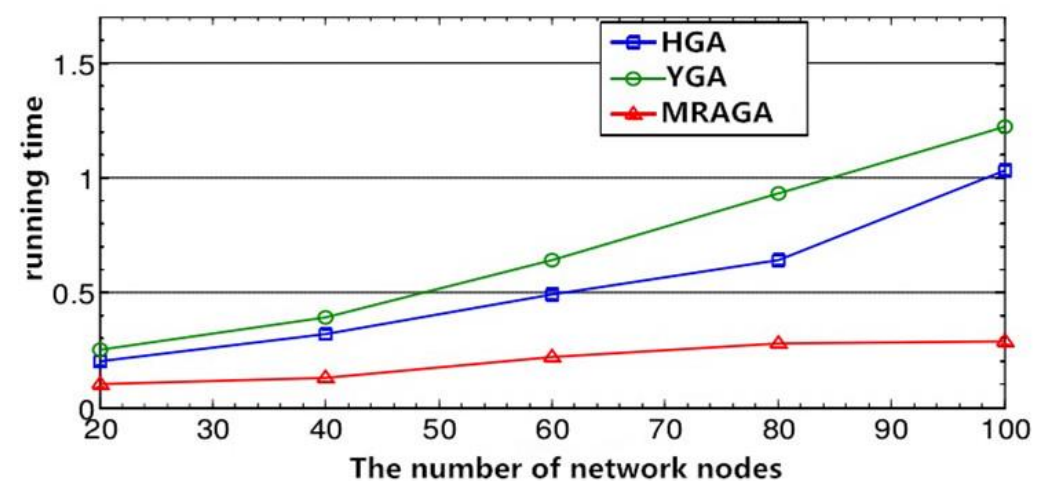

Figure 8. Comparison of Running Time (Multicast Group Size: 20\%-30\% Network Nodes)

\section{References}

[1] V. D. M. Schaar and D.S. Turaga, "Cross-layer packetization and retransmission strategies for delaysensitive wireless multimedia transmission”, IEEE Transactions on Multimedia, vol. 9, no.1, (2007), pp. 185-197.

[2] W. He, K. Nahrstedt and X. Liu, "End-to-end delay control of multimedia applications over multihop wireless links", ACM Transactions on Multimedia Computing, Communications, and Applications, vol. 5 , no. 2, (2008).

[3] S. Mahfoudh and P. Minet, "Maximization of energy efficiency in wireless ad hoc and sensor networks with SERENA", Mobile Information Systems, vol. 5, no. 1, (2009), pp. 33-52.

[4] M. Tarique and K.E. Tepe, "Minimum energy hierarchical dynamic source routing for mobile ad hoc networks", Ad Hoc Networks, vol. 7, no. 6, (2009), pp. 1125-1135.

[5] G. Anastasi, M. Conti, M.D. Francesco and A. Passarella, "Energy conservation in wireless sensor networks: a survey", Ad Hoc Networks, vol. 7, no. 3, (2009), pp. 537-568.

[6] S. Albers, "Energy-efficient algorithms", ACM Communications, vol. 53, no. 5, (2010), pp. 86-96.

[7] J.S. Lee, Y. Seunghwan and S.C.C. Kim, "Energy aware routing in location based ad-hoc networks", In Proc. ISCCSP, (2010), pp.1-5.

[8] P. Ruiz and P. Bouvry, "Enhanced distance based broadcasting protocol with reduced energy consumption”, In Proc. HPCS, (2010), pp.249-258.

[9] E. Gelenbe and C. Morfopoulou, "A framework for energy-aware routing in packet networks", The Computer Journal, vol. 54, no. 6, (2011), pp. 850-859.

[10] A. Jain, S.R. Kulkarni and S. Verdú, "Minimum energy per bit for wideband wireless multicasting: performance of decode-and-forward", In Proc. IEEE INFOCOM, (2010), pp.1-9. 
[11] S.C. Hu, T.H. Huang, A.C. Pang and W.H. Chen, "Fully localized energy-efficient multicast in largescale wireless ad hoc networks", International Journal of Ad Hoc and Ubiquitous Computing, vol. 7, no. 2, (2011), pp.100-111.

[12] A. Singh and W.N. Bhukya, "A hybrid genetic algorithm for the minimum energy broadcast problem in wireless ad hoc networks", Applied Soft Computing, vol. 11, no. 1, (2011), pp. 667-674.

[13] Y. Cai and L. Zhang, "Minimum-energy multicast using random llinear network coding", In Proc. MSN, (2011), pp.16-18.

[14] S. Guo and O.W.W. Yang, "Energy-aware multicasting in wireless ad hoc networks: a survey and discussion", Computer Communications, vol. 30, no. 9, (2007), pp. 2129-2148.

[15] A.J. Goldsmith and S.B. Wicker, "Design challenges for energy-constrained ad hoc wireless networks.", IEEE Wireless Communications, vol. 9, no. 4, (2002), pp. 8-27.

[16] A.T. Haghighat, K. Faez, M. Dehghan, A. Mowlaei and Y. Ghahremani, "A genetic algorithm for Steiner tree Optimization with multiple constraints using prüfer number”, In Proc. ICT, (2002), pp.272280.

[17] Y.S. Yen, Y.K. Chan, H.C. Chao and J.H. Park, "A genetic algorithm for energy-efficient based multicast routing on MANETs", Computer Communications, vol. 31, no. 4, (2008), pp. 858-869.

[18] M. Wall, "GAlib: a C++ library of genetic algorithm components", http://lancet.mit.edu/ga, (1996).

\section{Author}

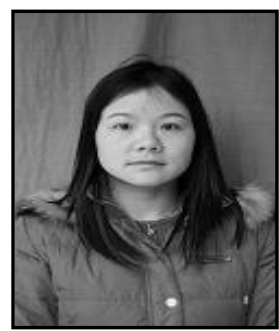

Yanhua Chen, she received her B.S degree from Sichuan Normal University. She received her M.S degree from Sichuan Normal University. She is a senior experimenter in Modern education technology center of Neijiang Normal University. Her research interests include Computer Application. 